\title{
Interdisziplinäre Therapie beim SCCHN
}

- Die leitliniengerechte Betreuung von Patienten mit Kopf-Hals-Tumoren (SCCHN) erfordert eine intensive interdisziplinäre Zusammenarbeit der beteiligten Fächer. Das betonte Prof. Dr. Ulrich Keilholz von der Charité, Berlin. An Organzentren sollte eine interdisziplinäre Studiengruppe die Therapie bestimmen. Als Beispiel führte Prof. Dr. Andreas Dietz vom Universitätsklinikum Leipzig die Kombinationstherapie aus Strahlentherapie (RT) und Cetuximab (Erbitux ${ }^{\circledR}$ ) an, die beim lokal fortgeschrittenen SCCHN einen Überlebensvorteil nach 5 Jahren von $9,2 \%$ bietet.

Ebenso lassen sich durch die Kombination von sequentieller Radiochemotherapie mit Cetuximab (statt Cisplatin) die Ergebnisse deutlich verbessern, weil die Patienten unter Cetuximab die Strahlendosis eher tolerieren. Das zeigte sich in der TREMPLINStudie an 153 Patienten im Stadium III-IV mit Larynx- oder Hypopharynx-Ca., die Dietz referierte [Lefebvre J et al. ASCO 2009, Abstr. $6010]$. Hier konnten $71 \%$ der Patienten unter
Cetuximab die volle Strahlendosis erhalten, unter Cisplatin $45 \%$. Die guten Studienergebnisse mit Cetuximab gingen auch in die aktuelle ESMO-Leitlinie für die Erstlinienbehandlung von rezidivierten und/oder metastasierten Kopf-Hals-Tumoren (Kombination mit Cisplatin) sowie für die lokal fortgeschrittenen SCCHN (Kombination mit RT) ein.

Schwierig ist bei diesen Patienten noch die individualisierte Therapie, da hier der Erfolg von Cetuximab nicht von der Zahl der EGFRGenkopien abhängt, so Keilholz. Lediglich die typische Hautreaktion scheint einen Hinweis auf den Erfolg des Biologics zu erlauben.

tuk

Satellitensymposium „Interdisziplinäre Zusammenarbeit in der Therapie von Kopf-HalsTumoren" im Rahmen des 29. Deutschen Krebskongresses. Berlin, 25. Februar 2010. Veranstalter: Merck Serono, Darmstadt

\section{Physikalischer Schleimlöser in neuer Dosis}

— Zu den üblichen Begleiterscheinungen von Atemwegserkrankungen wie Sinusitis, Bronchitis, Pseudokrupp oder Pneumonie gehört die Bildung von viskösem Sputum. Seit Jahrzehnten wird zur sekretolytischen Begleittherapie verschiedenartiger Atemwegserkrankungen deshalb Tacholiquin ${ }^{\circledR}$ eingesetzt. Das Mukolytikum ist in zwei Konzentrationen erhältlich: als 1\%ige und $0,1 \%$ ige Lösung. Bisher gab es sie nur in Flaschen von 20 und $30 \mathrm{ml}$, neuerdings ist Tacholiquin nun auch als 5-ml-Monodosis erhältlich und kann mit verschiedenen Therapeutika wie etwa Antibiotika oder Kortikoiden gemischt werden. Eine Packung der neuen Monodosen umfasst jeweils 10 Einheiten.

Seine Wirkung erzielt das Präparat rein physikalisch, der Schleim wird allein durch eine verringerte Oberflächenspannung gelöst. Aufgrund der physikalischen Wirkweise eignet es sich für alle Altersgruppen und kann auch schon bei Frühgeborenen und Säuglingen eingesetzt werden. In der Regel wird die Lösung mit einem Inhalationsgerät

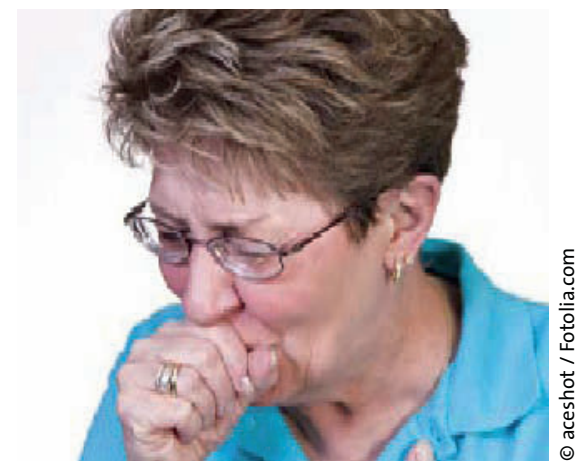

Mukolyse ist ein wichtiger Therapiebaustein bei vielen Atemwegserkrankungen

vernebelt. Die dabei entstehenden feinsten Tröpfchen gelangen in die oberen und unteren Atemwege. Durch Fachpersonal kann Tacholiquin ${ }^{\circledR}$ im Bedarfsfall direkt instilliert werden.

gz

Nach Informationen von bene Arzneimittel, München 